\title{
SEROPREVALENCIA DE LA DIROFILARIOSIS Y EHRLICHIOSIS CANINA EN TRES DISTRITOS DE LIMA
}

\author{
Jorge Adrianzén G. ${ }^{1}$, Amanda Chávez V.,3, Eva Casas A. ${ }^{2}$ y Olga Li E. ${ }^{4}$
}

\section{Abstract}

Canine Heartworm Disease is a parasitic disease caused by the nematode Dirofilaria immitis. The adult forms are found mainly in the pulmonary arteries and the right heart of canids. This nematode is transmitted by intermediate hosts which are blood suckling mosquitoes. Canine Ehrlichiosis is a disease of domestic and wild canids, caused by the rickettsia Ehrlichia canis, that infects mononuclear cells. This microorganism is transmitted by an arthropod vector, the brown dog-tick Rhipicephalus sanguineus. The objective of this assay was to determine the prevalence of Dirofilaria immitis and Ehrlichia canis in dogs of Chorrillos, La Molina and San Juan de Miraflores districts, which are adjacent to naturally stagnated waters. Blood samples were taken from 140 dogs chosen randomly without regard of breed, age and sex from February to May, 2001. The diagnosis was made by the detection of the D.immitis antigen and antibodies against E. canis, using a commercial ELISA kit. The results showed an expected prevalence of $4.4 \%$ for D. immitis and $16.5 \%$ for $E$. canis. This study is the first report detecting antibodies against $E$. canis in Peru.

Key words: Dirofilaria immitis, Ehrlichia canis, seroprevalence, ELISA test, antigen

\section{RESUMIEN}

La Dirofilariosis Canina es una enfermedad parasitaria, provocada por el nemátode Dirofilaria immitis. Sus formas adultas se alojan principalmente en las arterias pulmonares y en la parte derecha del corazón de los caninos, y se transmite por hospederos intermediarios, mayormente mosquitos hematófagos. La Ehrlichiosis Canina es una enfermedad de los caninos, cuyo agente etiológico es la rickettsia Ehrlichia canis que infecta intracitoplasmáticamente a los monocitos circulantes, y se transmite por un artrópodo vector, "la garrapata marrón del perro" Rhipicephalus sanguineus. El objetivo de este estudio fue determinar la prevalencia de Dirofilaria immitis y Ehrlichia canis en los distritos de Chorrillos, La Molina y San Juan de Miraflores, que son colindantes con aguas naturalmente estancadas. Se recolectaron muestras de sangre de 140 caninos al azar sin distinción de raza, edad y sexo, durante los meses de febrero a mayo del 2001. Se detectó el antígeno de $D$. immitis y los anticuerpos contra $E$. canis mediante la técnica de ELISA, utilizando un kit comercial. Se obtuvo una prevalencia de $4.4 \%$ para D. immitis y $16.5 \%$ para $E$. canis, demostrando que la seroprevalencia de $D$. immitis permanece constante en Lima. Este estudio reporta por primera vez en el país anticuerpos contra E. canis.

Palabras clave: Dirofilaria immitis, Ehrlichia canis, seroprevalencia, prueba de ELISA, antígeno

\footnotetext{
${ }^{1}$ Práctica privada

${ }^{2}$ Laboratorio de Microbiología y Parasitología Veterinaria, FMV-UNMSM

${ }^{3}$ E-mail: a_chavez_g@hotmail.com

${ }^{4}$ Laboratorio de Patología Clínica Veterinaria, FMV-UNMSM
} 


\section{INTRODUCCIÓN}

La dirofilariosis canina es una enfermedad parasitaria, de curso generalmente crónico, provocada por el nemátode Dirofilaria immitis. En su ciclo de vida intervienen hospederos intermediarios, los cuales son principalmente mosquitos hematófagos. Las formas adultas de $D$. immitis se alojan mayormente a nivel de las arterias pulmonares y la parte derecha del corazón de los caninos y felinos, pudiendo también infectar al hombre. En casos más severos, obstruyen las venas cavas, principalmente la posterior, existiendo hallazgos de formas adultas en las venas hepáticas (Grubissich, 1999).

Esta enfermedad tiene una distribución mundial, y se ve favorecida cuando tiene una población de hospederos susceptibles, un reservorio estable para la enfermedad, una población estable de especies del vector competente, y un clima propicio para el desarrollo del parásito (Johnstone et al., 1997).

La importancia de este estudio radica en la posibilidad de que se incremente la prevalencia de estas parasitosis en el Perú debido al aumento de la importación de perros provenientes de países con altas tasas de infección como España $(84.6 \%$, Cancrini et al., 2000), EEUU (60\%, Ryan y Newcomb, 1996), Japón (59\%, Companion Animal Surgery, 1997) y Argentina (60\%, Grubissich, 1999). Asimismo los perros que viven en zonas aledañas a aguas estancadas, como es el caso de los Pantanos de Villa en Chorrillos, Rinconada del Lago en La Molina y las lagunas de oxidación en San Juan de Miraflores tienen un mayor riesgo de adquirir la dirofilariosis por ser ambientes favorables para el desarrollo de las formas larvarias del mosquito vector.

La ehrlichiosis canina es una enfermedad inmunodepresiva de los caninos domésticos, silvestres y de distribución mundial. Es también llamada "enfermedad del perro rastreador", "pancitopenia canina tropical", "fiebre canina hemorrágica', y "tifus canina" (Frisby, 1997). Esta enfermedad es producida por la Ehrlichia canis, microorganismo gram negativo, pleomórfico, de la familia de las Rickettsiaceae, que infecta a los monocitos circulantes dentro de su citoplasma en agregados llamados 'mórulas'. E. canis es transmitida por la garrapata marrón del perro, Rhipicephalus sanguineus, la cual ocurre en forma transestadial, pero no transovárica (Waner y Harrus, 2000). La infección dentro del animal se disemina vía sanguínea o linfática dentro de las células mononucleares infectadas, llegando a otros sistemas orgánicos (Tesouro y Sainz, 1993). La mayoría de casos se presenta en áreas endémicas durante los meses de primavera y verano, cuando la población de garrapatas es más activa. Como la trasmisión de la ehrlichiosis es mecánica y no biológica, las transfusiones de sangre infectada pueden ocasionar altas tasas de infección (Waner y Harrus, 2000). La garrapata marrón del perro, $R$. sanguineus, tiene una alta prevalencia en el Perú (Liberato, 1998; Bustamante, 1998; Estares, 1999); sin embargo, no existen reportes sobre la presencia de la $E$. canis en el Perú.

El objetivo de este estudio fue determinar la prevalencia de D. immitis y E. canis en caninos aparentemente sanos en áreas colindantes con aguas naturalmente estancadas en tres distritos de Lima, a través de un kit comercial de ELISA.

\section{Materiales y Métodos}

El estudio se realizó en las áreas de los Pantanos de Villa en Chorrillos, en Rinconada del Lago en La Molina y en las lagunas de oxidación del distrito de San Juan de Miraflores, entre los meses de febrero a mayo del 2001. Estas zonas se caracterizan por tener áreas con aguas naturalmente estancadas.

El tamaño de la población a muestrear para dirofilariosis se calculó en base a la prevalencia hallada por Bravo (2001), utilizando 
la fórmula de proporción en poblaciones infinitas, con nivel de confianza del $95 \%$ y un error máximo permisible de $5 \%$. Se determinó un mínimo de 101 animales.

En el caso de ehrlichiosis canina no se disponía de datos previos por lo que se realizó un muestreo inicial con 30 perros, encontrándose a tres animales positivos. En base a esto se calculó el tamaño de la población a muestrear utilizando la misma fórmula, determinándose un mínimo de 139 animales.

\section{Procesamiento de muestras}

Se colectó 140 muestras de sangre en perros seleccionados al azar, sin distinción de raza, sexo y edad, utilizando vacutainers con anticoagulante EDTA. Además se anotó las horas de permanencia del animal dentro de la casa del propietario. Las muestras se llevaron refrigeradas hasta el Laboratorio de Parasitología de la Facultad de Medicina Veterinaria de la Universidad Nacional Mayor de San Marcos donde se les extrajo el plasma y se almacenó en congelación a $-20^{\circ} \mathrm{C}$ hasta su análisis.

Se utilizó el kit comercial de ELISA "IDEXX" Snap Combo Canino. Esta prueba enzimática puede detectar simultáneamente antígeno de excreción y secreción de $D$. immitis y anticuerpo (IgG) para E. canis.

\section{Análisis estadístico}

Se calculó la prevalencia aparente (proporción de animales infectados de acuerdo al número de animales positivos). Sin embargo, para determinar la prevalencia real se obtuvo la prevalencia corregida, tomando en cuenta la sensibilidad y especificidad de la prueba para cada agente (D. immitis: especificidad $=100 \%$; sensibilidad $=98.9 \%$ y para $E$. canis: especificidad $=100 \%$; sensibilidad $=95 \%$ ).

Se determinó el intervalo de confianza del 95\% que contiene al parámetro estimado. Asimismo, el efecto de las variables dis- trito, sexo, edad y horas de permanencia en el hogar sobre la cantidad de animales positivos se evaluó mediante la prueba de regresión logística.

\section{Resultados}

La prevalencia de Dirofilaria immitis y Ehrlichia canis en tres distritos colindantes con áreas de aguas naturalmente estancadas determinada entre los meses de febrero a mayo del 2001 fue de $4.4 \pm 3.4$ y $16.5 \%$ \pm 6.2 , respectivamente (Cuadro 1 ).

La prueba de regresión logística para D. immitis muestra que no existen diferencias estadísticas para las variables de distrito, edad, permanencia en el hogar y sexo. Sin embargo, para el caso de E. canis se pudo demostrar que si bien no existen diferencias estadísticas para las variables de distrito y edad, se encontraron diferencias estadísticas significativas para las variables de permanencia en el hogar y sexo. Es así que los animales que permanecen más tiempo fuera del hogar y que son hembras tienen mayor riesgo de adquirir la enfermedad

\section{Discusión}

Los primeros estudios sobre dirofilariosis en Lima Metropolitana fueron realizados utilizando la técnica de Knott modificado. Así, Hernández (1958) reportó un 8.8\% de prevalencia, en tanto que Bellido (1995) encuentra el $2.0 \%$ de prevalencia en perros de criaderos. Resultados similares fueron obtenidos por Acha (1952) con 100 perros de necropsia en la zona de Lima. A partir del 2000 se inician los primeros estudios serológicos de esta enfermedad utilizando una prueba comercial de ELISA indirecta en animales aparentemente sanos, encontrándose el $7.3 \%$ en la ribera del río Lurín (Bravo, 2001), 3\% en la ribera del río Chillón (Chipana, 2001) y $4.7 \%$ en la ribera del río Rímac (Acuña, 2002). Los resultados obtenidos en 
Cuadro 1. Presencia de Dirofilaria immitis y Ehrlichia canis mediante la prueba de ELISA, en distritos colindantes con aguas naturalmente estancadas (Chorrillos, La Molina y San Juan de Miraflores). Febrero - mayo, 2001

\begin{tabular}{|c|c|c|c|c|c|}
\hline \multirow{2}{*}{ Distritos } & \multirow{2}{*}{$\begin{array}{c}\text { Animales } \\
\text { muestreados }\end{array}$} & \multicolumn{2}{|c|}{ D. immitis } & \multicolumn{2}{|c|}{ E. canis } \\
\hline & & $\mathrm{N}^{\circ}$ positivos & $\% \pm \mathrm{IC}^{1}$ & $\mathrm{~N}^{\mathrm{o}}$ positivos & $\% \pm \mathrm{IC}$ \\
\hline Chorrillos & 57 & 3 & $5.3 \pm 5.8$ & 11 & $19.3 \pm 10.3$ \\
\hline La Molina & 23 & 0 & 0 & 2 & 8.7 \\
\hline San Juan de M. & 60 & 3 & $5.0 \pm 5.5$ & 9 & $15.0 \pm 9.0$ \\
\hline Total & 140 & 6 & $4.4 \pm 3.4$ & 22 & $16.5 \pm 6.2$ \\
\hline
\end{tabular}

${ }^{1}$ Intervalo de confianza del 95\%

el presente estudio son similares a los reportados en estos últimos trabajos, con lo cual se confirmaría la presentación constante de esta parasitosis.

En la prueba de regresión logística para D. immitis no se encontraron diferencias significativas para la variable permanencia en el hogar; sin embargo, se determinó que todos los animales positivos permanecían el mayor tiempo del día fuera de las casas de sus dueños. Asimismo, la ausencia de animales positivos en La Molina podría explicarse por el tipo de crianza, ya que la mayoría de estos animales permanecen dentro de las casas, por lo que no estarían expuestos a las picaduras de los mosquitos. Además, estos animales viven en hogares de estrato socioeconómico alto, por lo cual se podría presumir que reciben un control sanitario adecuado, entre ello, el uso de lactonas macrocíclicas para el control de diferentes parásitos; y este grupo químico puede afectar a las microfilarias actuando también como tratamiento preventivo para dirofilariosis.

Así mismo, la prevalencia de la ehrlichiosis en el distrito de La Molina fue la más baja $(8.7 \%)$, con respecto a los distritos de Chorrillos y San Juan de Miraflores (19.3 y $15.0 \%$, respectivamente), aunque sin dife- rencias estadísticas significativas entre distritos. Sin embargo, el bajo porcentaje en La Molina fue probablemente debido al mejor control sanitario de esos animales.

El $16.5 \%$ de perros positivos a ehrlichiosis constituye una cifra inicial de la situación de la ehrlichiosis canina en el Perú. Existen escasos estudios sobre E. canis en el continente; así, en el estado de Zulia en Venezuela se reporta un $83.6 \%$ de casos positivos en frotices sanguíneos coloreados (Arraga, 1992), y en Minas Gerais, Brasil se ha reportado un $16 \%$ de ehrlichiosis (Moreira et al., 2002).

En el análisis estadístico para E. canis se encontró diferencias significativas en las variables de permanencia en el hogar y sexo, teniendo mayor riesgo de infectarse los animales hembras y aquellos que pasan mayor tiempo del día fuera de sus hogares.

Los animales que permanecen más horas del día a la intemperie tienen mayor contacto con el medio ambiente, lugar donde las garrapatas vectoras realizan la muda entre sus estadíos, hecho que explicaría la diferencia encontrada. En cuanto a los animales hembras, se podría explicar que estos animales presentan situaciones donde su sistema 
inmune se encuentra disminuido, como en el momento del celo o la preñez; sin embargo, no se puede afirmar esta hipótesis hasta realizar más estudios sobre la enfermedad en nuestro medio y con mayor número de animales.

\section{ConClusiones}

- La seroprevalencia de Dirofilaria immitis y Ehrlichia canis fue de $4.4 \% \pm$ 3.4 IC y de $16.5 \% \pm 6.2$ IC, respectivamente, en caninos de distritos colindantes a zonas con aguas naturalmente estancadas (Chorrillos, La Molina y San Juan de Miraflores) y determinada con la prueba de ELISA comercial IDEXX.

- La dirofilariasis canina se encuentra dentro de los rangos esperados, y similar a estudios previos.

- Se deben realizar mayores estudios sobre la ehrlichiosis canina en otros distritos de Lima Metropolitana para conocer la real situación de esta enfermedad.

\section{LitTeratura Citada}

1. Acha, P. 1952. Porcentaje de parasitosis del Canis familiaris en la ciudad de Lima. Tesis de Bachillerato. Fac. Medicina Veterinaria, Univ. Nacional Mayor de San Marcos, Lima. 35 p.

2. Acuña, P. 2002. Determinación de la prevalencia de Dirofilaria immitis en los distritos de San Martín de Porres, Lima y Rímac. Tesis de Médico Veterinario. Fac. Medicina Veterinaria, Univ. Nacional Mayor de San Marcos, Lima. 47 p.

3. Arraga de Alvarado, C.M. 1992. Ehrlichiosis canina en Maracaibo, Estado Zulia, Venezuela. Reporte de 55 casos. Rev. Cient. Univ., Zulia 2: 30-40.

4. Bellido, M.E. 1995. Prevalencia de Dirofilaria immitis en criaderos de perros (Canis familiaris) en Lima Metro- politana. Tesis de Médico Veterinario. Fac. Medicina Veterinaria, Univ. Nacional Mayor de San Marcos, Lima. 38 p.

5. Bravo, $R$. 2001. Estudio de la dirofilariosis canina en los distritos de Lima de la ribera del río Lurín (Cieneguilla, Pachacámac y Lurín). Tesis de Bachillerato. Fac. Medicina Veterinaria, Univ. Nacional Mayor de San Marcos, Lima. 48 p.

6. Bustamante, A. 1998. Prevalencia de ectoparásitos en Canis familiaris en la zona climática litoral de Lima Metropolitana en la estación de invierno. Tesis de Bachillerato. Fac. Medicina Veterinaria, Univ. Nacional Mayor de San Marcos, Lima. 43 p.

7. Cancrini, G.; E. Allande; G Favia; F. Bornay; F. Antón; F. Simon. 2000. Canine dirofilariosis in two cities of southeastern Spain. Veterinary Parasitology 92: 81-86.

8. Chipana, C. 2001. Estudio de la dirofilariosis canina en los distritos de Lima de la ribera del río Chillón (Carabayllo, Comas, Los Olivos, Puente Piedra y Ventanilla). Tesis de Médico Veterinario. Fac. Medicina Veterinaria, Univ. Nacional Mayor de San Marcos, Lima. 45 p.

9. Companion Animal Surgery. 1997. Dirofilariasis - 'Heartworm'. [On Line] disponible: http://www.comvet.com.html/ heartworm.html [28/08/01].

10.Estares, L. 1999. Prevalencia de ectoparásitos de Canis familiaris en los distritos de San Juan de Lurigancho, San Martín de Porres, Comas e Independencia de Lima Metropolitana. Tesis de Médico Veterinario. Fac. Medicina Veterinaria, Univ. Nacional Mayor de San Marcos, Lima. $61 \mathrm{p}$.

11. Frisby, H. 1997. Ehrlichiosis. [OnLine] disponible: http://www.peteducation.com/ dogs/ehrlichia.htm [28/08/01].

12. Grubissich, J.A. 1999. Dirofilariasis canina. Rev. Holliday News 2: 8-12.

13. Hernández, A. 1958. Contribución al estudio de la filariasis canina en la ciu- 
dad de Lima. Tesis de Bachillerato. Fac. Medicina Veterinaria, Univ. Nacional Mayor de San Marcos, Lima. 29 p.

14. Johnstone, C; D.H. Knight; J.B. Lok. 1997. Parasitology - Dirofilaria immitis. [OnLine] disponible: http://cal.vet.upenn. edu/parasit/heartworm/hw-1.html [28/08/ $01]$.

15. Liberato, W. 1998. Prevalencia de ectoparásitos en Canis familiaris en los distritos de San Juan de Miraflores, Villa María del Triunfo y Villa el Salvador. Tesis de Bachillerato. Fac. Medicina Veterinaria, Univ. Nacional Mayor de San Marcos, Lima. $31 \mathrm{p}$.

16.Moreira, S.M.; C.V. Bastos; R.B. Araujo; M. Santos; L.M.F. Passos. 2002. Estudo retrospectivo (1998 a
2001) da erliquiose canina em Belo Horizonte, Minas Gerais, Brasil. Arquivo Brasileiro de Medicina Veterinária e Zootecnia 50: 20-25.

17. Ryan, W.; K. Newcomb. 1996. Prevalence of feline heartworm disease - A global review. Momentum Tech. MSD AGVET 3: 6-12.

18. Tesouro, M; A. Sainz. 1993. Ehrlichiosis canina. Veterinaria Información. Revisión del Consejo General de Colegios Veterinarios de España. No 132. p 114-119.

19. Waner, T.; S. Harrus. 2000. Canine Monocytic Ehrlichiosis. [On Line] disponible: http://www.ivis.org/advances/ Infect_Dis_carmichael/waner/chapter_frm.asp [28/12/01]. 